\title{
A localization computation in confining phase
}

\author{
Seiji Terashima \\ Yukawa Institute for Theoretical Physics, Kyoto University, \\ Kyoto, 606-8502 Japan \\ E-mail: terasima@yukawa.kyoto-u.ac.jp
}

ABSTRACT: In this note we show that the gaugino condensation of $4 \mathrm{~d} \mathcal{N}=1$ supersymmetric gauge theories in the confining phase can be computed by the localization technique with an appropriate choice of a supersymmetry generator.

KEYwords: Supersymmetric gauge theory, Nonperturbative Effects, Solitons Monopoles and Instantons, Supersymmetric Effective Theories

ArXiv EPRINT: 1410.3630 


\section{Contents}

1 Introduction and summary 1

$24 \mathrm{~d} \mathcal{N}=1$ SUSY gauge theory on $S^{1} \times S^{3}$ and localization 2

3 Gaugino condensation in SUSY Yang-Mills by the localization $\quad 7$

4 Including chiral multiplets $\quad 11$

A $4 \mathrm{~d} \mathcal{N}=1$ SUSY gauge theory on $S^{4} \quad 11$

\section{Introduction and summary}

An analytic computations in quantum field theories are usually hopeless except in some special classes of them. The supersymmetric (SUSY) gauge field theories are in the special class. The early example of such computations in the SUSY field theories is the Witten index [1]. Recently, the localization computations in the SUSY field theories on curved space initiated by Pestun [2] have been investigated intensively, which can be considered as generalizations of the topological field theories [3] and the Nekrasov partition function [4]. The localization computations use deformations of the action which do not change the partition function and some correlation functions and have been generalized to other $4 \mathrm{~d}$ manifolds [5]-[7] and also SUSY theories in various dimensions (see, for examples, [8]-[22]).

The other powerful technique to compute exactly some correlators in SUSY gauge theories is the holomorphy of the "superpotential" (see the review [23]). Indeed, Seiberg found the exact low energy superpotential of $4 \mathrm{~d} \mathcal{N}=1 \mathrm{SUSY} \operatorname{SU}\left(N_{c}\right)$ QCD on $\mathbf{R}^{4}[24]$ by using the holomorphy of the superpotential written in the superfields and the result of [25]. In particular, the gaugino condensation, i.e. the v.e.v. of the gaugino bi-linear, was exactly computed for the SUSY QCD with massive chiral multiplets. It was also computed in [26]. This technique was applied to the prepotential of $4 \mathrm{~d} \mathcal{N}=2$ SUSY gauge theories by Seiberg and Witten [27].

One might expect that the localization technique is powerful enough to compute any exactly computable quantity in SUSY gauge theories. For example, the Seiberg-Witten prepotential can be reproduced from the Nekrasov partition function. However, the above mentioned gaugino condensation is an obvious exception for this expectation so far. Indeed, the localization technique has not been applied to a SUSY gauge theory in the confining phase nor a computation of a v.e.v. of local operators like the gaugino bi-linear. Thus, it would be important and interesting to compute the gaugino condensation in $4 \mathrm{~d} \mathcal{N}=1$ SUSY SU $\left(N_{c}\right)$ (massive) QCD by the localization technique directly because this will open up other interesting applications of the technique. 
In this paper, we will show that the gaugino condensation can actually be computed by the localization technique with an appropriate choice of a SUSY generator. We consider the theory on $S^{1} \times \mathbf{R}^{3}$ and then take the limit to $\mathbf{R}^{4}$. ${ }^{1}$ The actual computation for this theory is almost identical to the one performed by Davies et al. [31-33], in which they consider the small radius limit of $S^{1}$ and argued that the gaugino condensation in the $\mathcal{N}=1$ SUSY Yang-Mills theory is independent of the radius of $S^{1}$. Furthermore, it was shown in [33] that the value of the gaugino condensation agrees with the one obtained in [24, 25] using the holomorphy. Therefore, we reproduce the gaugino condensation using the localization technique, ${ }^{2}$ and we hope that the discussions in the paper can be generalized to broader classes of SUSY gauge theories with only minor changes. Indeed, we believe that we can apply the arguments in this paper to generic $\mathcal{N}=1$ SUSY gauge theories and compute the chiral v.e.v. in the weak coupling limit. This is why we think the results in this paper are important although the explicit computations in the paper was essentially known as stated above.

The organization of this paper is as follows: in section 2 we first briefly review the $4 \mathrm{~d}$ $\mathcal{N}=1$ SUSY gauge theory on $S^{1} \times S^{3}$. Then, we show that by the localization technique the correlators of the lowest components of the chiral multiplets can be computed semiclassically around the anti-self-dual configurations. In section 3 we compute the gaugino condensation for the pure SUSY Yang-Mills on $S^{1} \times \mathbf{R}^{3}$ using the localization technique. We comment on the inclusion of the chiral multiplets shortly in section 4 . In the appendix, we construct the $4 \mathrm{~d} \mathcal{N}=1$ SUSY gauge theory on $S^{4}$ although the localization technique is not useful for it.

\section{$24 \mathrm{~d} \mathcal{N}=1$ SUSY gauge theory on $S^{1} \times S^{3}$ and localization}

In this section, we will briefly review the $4 \mathrm{~d} \mathcal{N}=1$ SUSY gauge theory on $S^{1} \times S^{3}[7$, $28,29,34]^{3}$ and then show that the path-integral for the correlation function of the lowest components of chiral multiplets can be reduced to the semi-classical computations around the instantons by adding an appropriate regulator action. Of course, the discussion in this section can be applied to the $4 \mathrm{~d} \mathcal{N}=1$ SUSY gauge theory on $S^{1} \times \mathbf{R}^{3}$ which is our main concern. Note that the SUSY theory with gaugino condensation can not be put on $S^{1} \times S^{3}$ without spontaneous breaking of the SUSY because of the required continuous

\footnotetext{
${ }^{1}$ Another simplest one is $4 \mathrm{~d} \mathcal{N}=1$ SUSY gauge theory on $S^{4}$ which we construct explicitly in the appendix. The computation of gaugino condensation of this theory would be related to the strong coupling instanton [30] because the instanton in $\mathbf{R}^{4}$ is related to the one in $S^{4}$. However, the localization technique can not be applied to this theory, at least naively, as we can see in the appendix. We will also argue that the direct application of the localization technique to the theory on $\mathbf{R}^{4}$ can not give the weak coupling limit.

${ }^{2}$ Precisely speaking, the localization computation can be done for $S^{1} \times \mathbf{R}^{3}$. However, the gaugino condensation of the theory on $\mathbf{R}^{4}$ can be obtained by the large radius limit. Note that the theory on $S^{1} \times \mathbf{R}^{3}$ is three dimensional theory in the IR limit, however, the scale is very small for the large radius. Thus, at a scale between this scale and the dynamical scale $\Lambda$ the theory is in a $4 \mathrm{~d}$ confining phase and the radius of $S^{1}$ cab be regarded as an infra-red regulator.

${ }^{3}$ We will closely follow the notations and conventions in [35].
} 
R-symmetry. ${ }^{4}$ However, there would be some $\mathcal{N}=1$ SUSY gauge theories on $S^{1} \times S^{3}$ which can have computable non trivial v.e.v. of the chiral multiplets by the localization technique. Thus, we will present the discussion in this section for the SUSY gauge theory on $S^{1} \times S^{3}$.

First, let us remind that the SUSY transformation of the vector multiplet for Euclidian $4 \mathrm{~d} \mathcal{N}=1$ SUSY gauge theory on $\mathbf{R}^{4}$ is

$$
\begin{aligned}
\delta A_{m} & =\frac{i}{2}\left(\epsilon \sigma_{m} \bar{\lambda}-\bar{\epsilon} \sigma_{m} \lambda\right), \\
\delta \lambda & =\frac{1}{2} \sigma^{m n} \epsilon F_{m n}-\epsilon D, \\
\delta \bar{\lambda} & =\frac{1}{2} \bar{\sigma}^{m n} \bar{\epsilon} F_{m n}-\bar{\epsilon} D, \\
\delta D & =-\frac{i}{2} \epsilon \sigma^{m} D_{m} \bar{\lambda}-\frac{i}{2} \bar{\epsilon}^{m} D_{m} \lambda,
\end{aligned}
$$

where $m=1,2,3,4$, all fields are in the adjoint representation of the gauge group $G$ and $\epsilon, \bar{\epsilon}$ are constant spinors. We have introduced the chiral decomposed Gamma matrix $\sigma^{m}$ : $\sigma^{a}$ is the Pauli matrix for $a=1,2,3$ and $\sigma^{4}=i$, and $\bar{\sigma}^{m}=\left(\sigma^{m}\right)^{\dagger}$. We also defined $\sigma_{m n}=\frac{1}{2}\left(\sigma_{m} \bar{\sigma}_{n}-\sigma_{n} \bar{\sigma}_{m}\right)$ and $\bar{\sigma}_{m n}=\frac{1}{2}\left(\bar{\sigma}_{m} \sigma_{n}-\bar{\sigma}_{n} \sigma_{m}\right)$. The spinor indices are raised or lowered by $\epsilon^{\alpha \beta}$ which has $\epsilon^{12}=-\epsilon_{12}=1 .^{5}$

It is known $[7,35]$ that the SUSY transformation (2.1) is consistent with the $4 \mathrm{~d} \mathcal{N}=1$ SUSY gauge theory on $S^{1} \times S^{3}$ if the derivative $D_{m}$ is defined as follows:

$$
D_{m}=\mathcal{D}_{m}-i q V_{m}
$$

where $\mathcal{D}_{m}$ is the covariant derivative including the spin connection, for example $\mathcal{D}_{m}=$ $\partial_{m}+\frac{1}{4} w_{m}^{a b} \gamma^{a b}$ for spinors. Here the $V_{m}$ is an appropriate background field and the $q$ is the R-charge for which we assigned $q(\epsilon)=1, q(\bar{\epsilon})=-1, q(\lambda)=1, q(\bar{\lambda})=-1$. For simplicity, we will consider the unit 3 -sphere and $d s^{2}=d t^{2}+d s_{S^{3}}^{2}$ where $0 \leq t<2 \pi R$ is the periodic coordinate for $S^{1}$. Then, the background field is fixed to be

$$
V_{m} d x^{m}=-\frac{i}{2} d t
$$

and the Killing spinors are given by the solutions of the following equations:

$$
D_{m} \epsilon=-\frac{1}{2} \sigma_{m} \bar{\sigma}_{4} \epsilon, \quad D_{m} \bar{\epsilon}=-\frac{1}{2} \bar{\sigma}_{m} \sigma_{4} \bar{\epsilon}
$$

It should be stressed that we regard $\lambda$ and $\bar{\lambda}$ are independent holomorphic fermionic 2 -components spinor fields in the $4 \mathrm{~d}$ Euclidian spacetime. Indeed, these two spinors are fundamental representations of the former and the latter $\mathrm{SU}(2)$ of $\mathrm{SU}(2) \times \mathrm{SU}(2)(=\mathrm{Spin}(4))$, thus they can not be related by the complex conjugation. Because any reality condition can not be imposed on the fundamental representation of $\mathrm{SU}(2)$, we regard $\lambda, \bar{\lambda}$ as the formal

\footnotetext{
${ }^{4}$ We thank an editor of a journal for pointing out this point.

${ }^{5}$ The normalizations of the $\sigma_{m n}, \bar{\sigma}_{m n}$ and the fermions are different from the one in the Wess-Bagger's text.
} 
holomorphic path-integral variables. The SUSY parameters $\epsilon$ and $\bar{\epsilon}$ are also independent fields.

Usual $\mathcal{N}=1$ SUSY Yang-Mills action with the theta term

$$
L_{g}=\operatorname{Tr}\left[\frac{1}{g^{2}}\left(\frac{1}{2} F_{m n} F^{m n}+D^{2}+i \bar{\lambda} \bar{\sigma}^{m} D_{m} \lambda\right)+i \frac{\theta}{16 \pi^{2}} F \tilde{F}\right]
$$

is SUSY invariant on $S^{1} \times S^{3}$ with the covariant derivative $D_{m}$ defined by (2.2). We will also use the complexified coupling constant $\tau \equiv \frac{\theta}{2 \pi}+\frac{4 \pi i}{g^{2}}$.

Now we are applying the localization technique to the theory. In this paper, the localization technique simply means the use of the following identity for the operators satisfying $\delta \mathcal{O}_{a}=0$ :

$$
\frac{d}{d t}\left\langle\mathcal{O}_{1} \mathcal{O}_{2} \cdots \mathcal{O}_{n} e^{-t \delta \int V}\right\rangle=0
$$

where $\delta$ is a symmetry transformation of the theory and $V$ should satisfies $\delta^{2} \int_{S^{3} \times S^{1}} V=0$. With this identity we can compute the correlator $\left\langle\mathcal{O}_{1} \mathcal{O}_{2} \cdots \mathcal{O}_{n}\right\rangle$ with a sufficiently large $t$ if the real part of $\delta \int_{S^{3} \times S^{1}} V$ is non-negative.

In order to use the localization technique, we should choose a SUSY transformation $\delta$ and a regulator Lagrangian $V$. Here the choice of $\delta$ means the choice of $\epsilon$ and $\bar{\epsilon}$. As in [2], we simply take the regulator Lagrangian as following:

$$
V=(\delta \lambda)^{\dagger} \lambda+(\delta \bar{\lambda})^{\dagger} \bar{\lambda}
$$

where we should define $(\delta \lambda)^{\dagger}$ appropriately.

First, let us assume both $\epsilon$ and $\bar{\epsilon}$ are nonzero. Then, the bosonic contributions from the first term is

$$
(\delta \lambda)^{\dagger} \delta \lambda \sim F_{m n}^{+} F^{+m n}+D^{2}
$$

where we have used

$$
\delta \lambda=\frac{1}{2} \sigma^{m n} \epsilon F_{m n}^{+}-\epsilon D
$$

and $F^{+}\left(F^{-}\right)$are the anti-self-dual (self-dual) part of the field strength $F$, respectively. Then, the saddle points for $t \rightarrow \infty$ will satisfy $F^{+}=0$ and $D=0$. Because the contribution from the other term in $V$, i.e. $(\delta \bar{\lambda})^{\dagger} \delta \bar{\lambda} \sim\left(F^{-}\right)_{m n}\left(F^{-}\right)^{m n}+D^{2}$, will give $F^{-}=0$, we conclude that the saddle points satisfy $F_{m n}=0$ and $D=0$ where the regulator action is essentially the Yang-Mills action. Thus the partition function can be calculated in the weak coupling limit. This is well-known result. Indeed, with the twist of the boundary condition along the $S^{1}$, the partition function on $S^{1} \times S^{3}$ is the superconformal index [7, 28, 29, 36, 37].

In this paper, we consider another possibility for the localization computation on $S^{1} \times$ $S^{3}$ : we take $\bar{\epsilon} \neq 0$, but $\epsilon=0$, which implies $\delta \delta=0$. Thus the condition $\delta \delta \int V=0$ is 
trivially satisfied. ${ }^{6}$ Explicitly, the SUSY transformation is

$$
\begin{aligned}
\delta A_{m} & =-\frac{i}{2} \bar{\epsilon} \sigma_{m} \lambda, \\
\delta \lambda & =0, \quad \delta \bar{\lambda}=\frac{1}{2} \bar{\sigma}^{m n} \bar{\epsilon} F_{m n}-\bar{\epsilon} D, \\
\delta D & =-\frac{i}{2} \bar{\epsilon} \bar{\sigma}^{m} D_{m} \lambda,
\end{aligned}
$$

With this $\delta$, we have $\delta \lambda=0$, thus a correlation function of any gauge invariant combinations of $\lambda$ can be computed by the localization technique. The Killing spinor can be taken explicitly as

$$
\bar{\epsilon}=\frac{1}{\sqrt{2}}\left(\begin{array}{c}
e^{\frac{i}{2}(-\chi+\phi+\theta)} \\
e^{\frac{i}{2}(-\chi+\phi-\theta)}
\end{array}\right),
$$

where we use the coordinate system with the metric $d s^{2}=\cos ^{2} \theta d \phi^{2}+\sin ^{2} \theta d \chi^{2}+d \theta^{2}$. Taking $V$ as in (2.7), we easily see that

$$
V=F_{m n}^{-} F^{-m n}+D^{2}=\frac{1}{2} F_{m n} F^{m n}+\frac{1}{2} F_{m n} \tilde{F}^{m n}+D^{2},
$$

where $\tilde{F}$ is the dual of the field strength, and the saddle points equations are $F^{-}=0$ and $D=0$, i.e. the instantons (anti-self-dual connections) on $S^{1} \times S^{3} \cdot{ }^{7}$ Note that this includes the Yang-Mills action with a pure imaginary $\theta$. This also means that by taking $t \rightarrow \infty$ limit the theory is arbitrary weak coupling because only the Yang-Mills action affects the fluctuations around the instantons. The value of the Lagrangian (2.5) at the saddle points is $2 \pi i \tau \times$ (instanton number) because $F=\tilde{F}$. It is interesting that there is a coupling constant dependence in the computation using the localization for the $\mathcal{N}=1$ SUSY gauge theory on $S^{1} \times S^{3}$.

For the chiral multiplets on $S^{1} \times S^{3}$, the SUSY transformation were given as

$$
\begin{aligned}
& \delta \phi=-\epsilon \psi, \quad \delta \psi=i \sigma^{m} \bar{\epsilon} D_{m} \phi+\frac{3 i r}{4} \sigma^{m} D_{m} \bar{\epsilon} \phi+\epsilon F, \\
& \delta \bar{\phi}=+\bar{\epsilon} \bar{\psi}, \quad \delta \bar{\psi}=i \bar{\sigma}^{m} \epsilon D_{m} \bar{\phi}+\frac{3 i r}{4} \bar{\sigma}^{m} D_{m} \epsilon \bar{\phi}+\bar{\epsilon} \bar{F}, \\
& \delta F=+i \bar{\epsilon} \bar{\sigma}^{m} D_{m} \psi+\frac{i(3 r-2)}{4} D_{m} \bar{\epsilon} \bar{\sigma}^{m} \psi-i \bar{\epsilon} \bar{\lambda} \psi, \\
& \delta \bar{F}=-i \epsilon \sigma^{m} D_{m} \bar{\psi}-\frac{i(3 r-2)}{4} D_{m} \epsilon \sigma^{m} \bar{\psi}-i \epsilon \bar{\psi} \lambda .
\end{aligned}
$$

where $r$ is the R-charge of $\phi$. Under the SUSY transformation, the following kinetic term is invariant:

$$
\begin{aligned}
\mathcal{L}_{m}= & D_{m} \bar{\phi} D^{m} \phi+\frac{1}{2}(3 r-2)\left(D_{4} \bar{\phi} \phi-\bar{\phi} D_{4} \phi\right)+\frac{3 r(-3 r+4)}{4} \bar{\phi} \phi-i \bar{\phi} D \phi \\
& -i \bar{\psi} \bar{\sigma}^{m} D_{m} \psi-i \frac{3 r-2}{2} \bar{\psi} \bar{\sigma}_{4} \psi+i \bar{\psi} \bar{\lambda} \phi+i \bar{\phi} \lambda \psi+\bar{F} F .
\end{aligned}
$$

\footnotetext{
${ }^{6}$ However, this means that we can not use the equivariant index theorem for the 1-loop computation even though the path-integral will be reduce to the semi-classical instanton calculation. The instantons do not localize to somewhere, for example, north or south poles.

${ }^{7}$ This saddle point equations was also noted in a recent paper [34].
} 
We can easily see that the usual superpotential terms are the SUSY invariant if the Rcharge is 2 .

For $\epsilon=0$, we have $\delta \phi=0, \delta \bar{F}=0$. Thus any gauge invariant combination of the lowest components of the chiral multiplets is invariant under the SUSY transformation generated by the $\delta$ with $\epsilon=0$. Now let us consider the following $\delta$-exact Lagrangian:

$$
L_{\bar{W}}=\delta\left(\frac{\partial \bar{W}(\bar{\phi})}{\partial \bar{\phi}^{i}} \bar{\eta} \bar{\psi}^{i}\right)
$$

where $\bar{\eta}$ is a Grassmann even spinor defined by

$$
\bar{\eta} \equiv U\left(\begin{array}{l}
0 \\
1
\end{array}\right)
$$

where

$$
U=\frac{1}{\sqrt{2}}\left(\begin{array}{cc}
e^{\frac{i}{2}(-\chi+\phi+\theta)} & -e^{-\frac{i}{2}(-\chi+\phi-\theta)} \\
e^{\frac{i}{2}(-\chi+\phi-\theta)} & e^{-\frac{i}{2}(-\chi+\phi+\theta)}
\end{array}\right),
$$

is an $\mathrm{SU}(2)$ matrix. Note that $\bar{\epsilon}=U\left(\begin{array}{l}1 \\ 0\end{array}\right)$ and then we have $\bar{\eta} \bar{\epsilon}=1$. This does not seem to keep the rotational symmetry of $S^{3}$ because of the presence of $\bar{\eta}$, however, this keeps it. Indeed, we can see that this Lagrangian is the anti-chiral part of the superpotential $W(\phi) .{ }^{8}$ Thus, the correlators we consider do not depend on parameters in the anti-chiral superpotential. This can be regarded as a derivation of the holomorphy without using the superfields.

Now we consider the following regulator Lagrangian:

$$
V=(\delta \psi)^{\dagger} \psi+(\delta \bar{\psi})^{\dagger} \bar{\psi}
$$

where the Hermite conjugate $(\dagger)$ is defined such that it is positive definite. We have

$$
V \sim\left|D_{m} \phi\right|^{2}+r^{2}|\phi|^{2}+|F|^{2}
$$

schematically. For a field with $r \neq 0$, the saddle point is trivial, i.e. $\phi=0$ and $F=0$. However, in the flat space limit $\left(S^{1} \times \mathbf{R}^{3}\right)$, we have

$$
V \sim\left|D_{m} \phi\right|^{2}+|F|^{2}
$$

and the saddle points are $D_{m} \phi=0$ and $F=0$. Therefore, in general the v.e.v. of the lowest components of the chiral multiplets can be computed by semi-classical computations if the theory are in the confining phase with SUSY. ${ }^{9}$

\footnotetext{
${ }^{8}$ For the fermionic part, we have$$
\frac{\partial \bar{W}(\bar{\phi})}{\partial \bar{\phi}^{i} \partial \bar{\phi}^{j}}\left(\bar{\epsilon} \bar{\psi}^{j}\right)\left(\bar{\eta} \bar{\psi}^{i}\right)=\frac{1}{2} \frac{\partial \bar{W}(\bar{\phi})}{\partial \bar{\phi}^{i} \partial \bar{\phi}^{j}}\left(\left(\bar{\epsilon} \bar{\psi}^{j}\right)\left(\bar{\eta} \bar{\psi}^{i}\right)+\left(\bar{\epsilon} \bar{\psi}^{i}\right)\left(\bar{\eta} \bar{\psi}^{j}\right)\right) \sim \frac{1}{2} \frac{\partial \bar{W}(\bar{\phi})}{\partial \bar{\phi}^{i} \partial \bar{\phi}^{j}}\left(\bar{\psi}^{j} \bar{\psi}^{i}\right)
$$

where the last equation can be shown by taking $\epsilon^{1}=1, \epsilon^{2}=0$. Thus the $L_{\bar{W}}$ is the anti-chiral part of the superpotential.

${ }^{9}$ Note that the regulator term (2.18) is a Kaehler potential and the (2.12) is the anti-chiral super potential which will not affect the v.e.v. of the lowest components of the chiral multiplets.
} 
As we stated at the beginning of this section, we need to find a SUSY theory with the non trivial v.e.v. of the chiral multiplets without breaking the SUSY on $S^{1} \times S^{3}$ in order to apply the discussion in this section. Furthermore, for an explicit computation of the correlation functions we need to construct the instantons on $S^{1} \times S^{3}$. Although it is important and interesting to perform these explicitly, we leave these problems in future. Instead, we will consider the $\mathcal{N}=1$ SUSY gauge theory on $S^{1} \times \mathbf{R}^{3}$ which is considered as a small curvature limit of $S^{1} \times S^{3}$.

\section{Gaugino condensation in SUSY Yang-Mills by the localization}

In this section, for simplicity, we will consider the $\mathcal{N}=1$ SUSY Yang-Mills theory with gauge group $\mathrm{G}$ which has a simple Lie algebra of rank $r$ on $S^{1} \times \mathbf{R}^{3}$ where the radius of $S^{1}$ is $R$. Here we require the periodic boundary conditions along $S^{1}$ for all fields. The action is given by (2.5). For this theory, the important correlation function which can be computed using the localization is the gaugino condensation

$$
\langle\operatorname{Tr}(\lambda \lambda)\rangle,
$$

which is known to be non-vanishing due to the strong coupling effects, i.e. the confinement effects. Indeed, this has been computed exactly for the theory on $\mathbf{R}^{\mathbf{4}}$ for the classical groups in $[26,38-40]$ which was also computed using the holomorphy of the superpotential. Furthermore, it was also computed by using the $R \rightarrow 0$ limit for any simple gauge group in [31, 32]. Although $R$ itself is not holomorphic variable, in [31, 32], it was argued that the gaugino condensation does not depend on $R|\Lambda|$ because of the holomorphy for the SUSY Yang-Mills theory and it is only the dimensionless combination the correlator can depend on.

Here, we would like to compute the gaugino condensation by using the localization technique. ${ }^{10}$ The computation we will see below is essentially same as the one in [31$33]$ and then the followings will be almost a brief review of [31-33]. However, we need to be careful to check that the validity of the computation in $R \rightarrow 0$ can be applied to our computation in $t \rightarrow \infty$ limit with $R$ fixed finite. We hope that our localization computation can be generalized to more general ones. In this section, we will use the notation and convention used in [33].

First, we will add the regulator action, whose bosonic part is $-t \int\left(\left(F^{-}\right)^{2}+D^{2}\right)$, to the original SUSY Yang-Mills action in order to compute $\langle\operatorname{Tr}(\lambda \lambda)\rangle$. Note that the path-integral measure is defined at $t=0$. Then, taking $t \rightarrow \infty$ limit, the path integral localized to the saddle point configurations which satisfy $F^{-}=0$ and $D=0$. Here, the 1-loop determinant

\footnotetext{
${ }^{10}$ For the field theory on a non-compact manifold, the weak coupling expansion for the $t \rightarrow \infty$ limit could not be valid by the IR effects. Indeed, if we consider the SUSY Yang-Mills on $\mathbf{R}^{4}$ and using the localization technique, the large $t$ will not mean the weak coupling. The effective dynamical scale determined by $t$ is very low in the large $t$ limit, however, the effective coupling of the $4 \mathrm{~d}$ spectrum below this scale is not weak coupling. On the other hand, we expect that the gauge theory on $S^{1} \times \mathbf{R}^{3}$ will be weak interacting in the large $t$ limit. In this case, below the mass scale $1 / R$ there are $3 \mathrm{~d}$ fields with non-zero v.e.v. which breaks the gauge group to the Abelian group, as we will see later. Thus, in this case we see that the theory is weak coupling in the large $t$ limit. Note that if we take $R \rightarrow \infty$, this breaking scale goes to zero.
} 
around the saddle points may be trivial because we are considering the flat space $\mathbf{R}^{3}$ with the Euclidian time. ${ }^{11}$ The saddle points (and every configurations) are characterized by the Wilson loop along $S^{1}$, the instanton charge and the magnetic charges. The Wilson loop is defined by

$$
\langle\phi\rangle=\lim _{\left|x^{\mu}\right| \rightarrow \infty} \int_{0}^{2 \pi R} d x_{0} A_{0},
$$

which should not be path-integrated and considered as a moduli of the vacua because we consider the theory on $S^{1} \times \mathbf{R}^{3}$ which is non-compact for the three directions. Note also that the instanton number $k$ is not necessary integer because we consider non-compact manifold. Assuming the generic non-zero Wilson loop, we have the decomposition of gauge group $G$ to $\mathrm{U}(1)^{r}$ which enable us to define the $r$ different magnetic charges. Among the saddle point configurations, we need the configurations which have precisely two zero modes of the fermions $\lambda$ under the anti-self-dual configurations in order to give a non-zero contribution to the gaugino condensation because it is a bi-linear of the fermions. These configurations are identified and are called the fundamental monopoles in [31-33]. These fundamental monopoles are usual BPS magnetic monopoles parameterized by $i=1, \cdots, r$ which is related to the embedding of $\mathrm{SU}(2)$ into $G$, and the $\mathrm{KK}$ monopole of [43]. It is known that the 1-instanton without magnetic charges on $S^{1} \times \mathbf{R}^{3}$ can be considered as a bound state of these $r+1$ monopoles.

The BPS magnetic monopole has magnetic charge $g$ given by $\alpha_{i}^{*}$ where $\alpha_{i}$ is a simple root. The instanton charge $k$ of it is $\frac{1}{2 \pi} \alpha_{i}^{*} \cdot\langle\phi\rangle$ and then the classical action is

$$
S=-i \tau \alpha_{i}^{*} \cdot\langle\phi\rangle
$$

Here $\alpha^{*} \equiv 2 \alpha /(\alpha \cdot \alpha)$. For the saddle points, the regularized action vanishes by definition, of course. For the KK monopole, we have $g=\alpha_{0}^{*}, k=1+\frac{1}{2 \pi} \alpha_{0}^{*} \cdot\langle\phi\rangle$, and

$$
S=-2 \pi i \tau-i \tau \alpha_{0}^{*} \cdot\langle\phi\rangle,
$$

where $\alpha_{0}$ is the lowest root which satisfies $\sum_{i=0}^{r} k_{i}^{*} \alpha_{i}^{*}=0$ with $k_{i}^{*}$ is the Kac labels.

The two fermionic zero modes are given by the SUSY transformation

$$
\lambda_{\alpha}=\sigma_{\alpha}^{m n \beta} \xi_{\beta} F_{m n} \rightarrow 4 \pi\left(S_{\mathrm{F}} \xi\right)_{\alpha} \alpha \cdot H
$$

where the limit means $\left|x_{\mu}\right| \rightarrow \infty$ and $S_{\mathrm{F}}=\sigma_{\mu} x^{\mu} /\left(4 \pi\left|x_{\nu}\right|^{3}\right)$ is the massless fermion propagator in $3 \mathrm{~d}$.

Before computing the correlators, we need to find the quantum vacua of the theory. Here we will find the quantum vacua and compute the gaugino condensation at $t \rightarrow \infty$ limit where the theory is weak coupling. Later, we will consider the original theory at $t=0$.

The classical massless fields for the $t \rightarrow \infty$ limit should be $\mathrm{U}(1)^{r}$ Abelian multiplets with zero KK momentum of $S^{1}$, which can be regarded as 3d fields. Because in the $t \rightarrow \infty$

\footnotetext{
${ }^{11}$ This is not precisely true. As shown in $[41,42]$, the 1-loop determinant is non-trivial because of the asymptotic behaviors of the monopoles, however, it only contributes to the Kähler potential. Thus, for the computation of the gauge condensation, it can be ignored.
} 
limit the zero modes and non-zero modes are decoupled each other, we can forget about the non-zero KK momentum modes. Thus the bosonic part of them are the Wilson loop scalars $\phi$ and dual photon scalars $\sigma$, which can be combined to $r$ complex scalars

$$
z=i(\tau \phi+\sigma) .
$$

Thus the $z$ are the classical moduli of vacua. By checking the SUSY transformations, we can see that this $z$ forms the $\mathcal{N}=1$ chiral multiplets $X$ with a rescaled massless fermions $\psi=2^{\frac{5}{2}} \pi^{2} \frac{R}{g^{2}} \lambda$, where we integrated out the auxiliary fields $D$ and have considered the on-shell multiplets. The kinetic terms are given by the original action as

$$
S=\left.\frac{1}{8 \pi R^{2}} \int d^{3} x \frac{1}{\operatorname{Im} \tau} X^{\dagger} X\right|_{\theta \theta \bar{\theta} \bar{\theta}},
$$

where no potential terms appears.

Now we will compute the scalar potential semi-classically and determine the vacua. Note that our regularization term is the SUSY Yang-Mills term with the non-zero theta term, thus it is invariant under all SUSY transformations. Therefore, instead of the scalar potential, we will compute the fermion bi-linear terms which are related to the superpotential and the scalar potential by the SUSY transformation. The fermion bi-linear can be non-zero only for the fundamental monopole configurations, on which the original action is evaluated to the following value:

$$
S_{j}=-2 \pi i \tau \delta_{j 0}-\alpha_{j}^{*} \cdot\langle z\rangle,
$$

for the $j$-th fundamental monopole. Note that the v.e.v. of the dual photon $\sigma$ also contributes to it because the additional action $\frac{i}{4 \pi} \int d^{3} x \epsilon_{\mu \nu \rho} \sigma \partial_{\mu} F_{\nu \rho}$ which forces the Bianchi identity gives the boundary term:

$$
\frac{i}{2 \pi} \int_{S^{2}=\partial\left(\mathbf{R}^{3}\right)} d x_{\mu} \sigma B_{\mu}
$$

The path-integral measure of the zero modes of the $j$-th monopole is given by

$$
\int d \mu_{\mathrm{mono}}^{(j)}=\frac{2}{\left(\alpha_{j}\right)^{2}} \frac{\mu^{3} R}{g^{2}} e^{-S_{j}} \int d^{3} a d \Omega d^{2} \xi
$$

where $a_{\mu}$ is the position on $\mathbf{R}^{3}, \Omega$ is the $\mathrm{U}(1)$ phase and $\xi$ is the Grassmann odd zero modes. Note that this includes the cut-off scale $\mu$ and the gauge coupling $g$. These two are defined by the original theory, especially $g$ is defined by the original action because the path-integral measure is defined at the original action, i.e. $t=0$. Using this measure and the asymptotic form of the fermionic zero modes, we have

$$
\left\langle\lambda_{\alpha}(x) \otimes \lambda_{\beta}(0)\right\rangle \sim \frac{2^{6} \pi^{3} \mu^{3} R}{g^{2}\left(\alpha_{j}\right)^{2}} \alpha_{j}^{*} \otimes \alpha_{j}^{*} e^{-S_{j}} \int d^{3} a S_{\mathrm{F}}(x-a)_{\alpha}^{\gamma} S_{\mathrm{F}}(a)_{\beta \gamma},
$$

for $|x| \rightarrow \infty$. The superpotential which gives the contributions of $r+1$ fundamental monopoles is found to be

$$
W(X)=\frac{2 \pi \mu^{3} R}{g^{2}}\left(\sum_{j=1}^{r} \frac{2}{\left(\alpha_{j}\right)^{2}} e^{\alpha_{j}^{*} X}+\frac{2}{\left(\alpha_{0}\right)^{2}} e^{2 \pi i \tau+\alpha_{0}^{*} X}\right) .
$$


The vacua can be fixed by $\frac{d W}{d X}=0$ to

$$
X=\sum_{j=1}^{r} a_{j} w_{j}, \quad \text { where } \quad e^{a_{j}}=\frac{k_{j}^{*}\left(\alpha_{j}\right)^{2} e^{2 \pi i \tau}}{2 \kappa},
$$

and

$$
\kappa^{c_{2}}=e^{2 \pi i\left(c_{2}-1\right) \tau} \prod_{j=0}^{r}\left(\frac{k_{j}^{*}\left(\alpha_{j}\right)^{2}}{2}\right)^{k_{j}^{*}},
$$

which has $c_{2}$ roots corresponding to $c_{2}$ vacua. Here $c_{2}=\sum_{i=0}^{r} k_{i}^{*}$ is the dual Coxeter number.

We can compute the gaugino condensation by evaluating the (3.11) by the fermion zero modes without taking the asymptotic form. More conveniently, we can use the relation

$$
\left\langle\frac{\operatorname{Tr} \lambda^{2}}{16 \pi^{2}}\right\rangle=\frac{1}{b_{0}} \Lambda \frac{\partial}{\partial \Lambda}\left\langle\frac{1}{2 \pi R} W\right\rangle
$$

which can be derived by uplifting the $\tau$ to a superfield and $\Lambda$ is the dynamical scale in the Pauli-Villars renormalization scheme at 2-loop order,

$$
\Lambda^{3}=\mu^{3} \frac{1}{g^{2}(\mu)} \exp \frac{2 \pi \tau(\mu)}{c_{2}},
$$

and $b_{0}=3 c_{2}$. Using this relation, we finally find

$$
\left\langle\frac{\operatorname{Tr} \lambda^{2}}{16 \pi^{2}}\right\rangle=\frac{\Lambda^{3} e^{2 \pi i u / c_{2}}}{\prod_{j=0}^{r}\left(k_{j}^{*}\left(\alpha_{j}\right)^{2} / 2\right)^{k_{j}^{*} / 2}},
$$

which does not depend on $R$. For example, $\left\langle\frac{\operatorname{Tr} \lambda^{2}}{16 \pi^{2}}\right\rangle= \pm \Lambda^{3}$ for $G=\operatorname{SU}(2)$.

Now we will consider the original theory at $t=0$. We will denote $\langle(\cdots)\rangle_{t}$ as the correlator of the original theory, but choosing the vacuum (or the boundary condition at spatial infinity) as the one of the theory with the regulator action with $t$. Note that the vacua of the theory deformed by the regulator action is not need to be the vacua of the original theory in general. Then, we have

$$
\frac{\partial}{\partial t}\left\langle\mathcal{O}_{1} \cdots \mathcal{O}_{m} e^{-t \int \delta V}\right\rangle_{t}=\lim _{\Delta t \rightarrow 0} \frac{1}{\Delta t}\left(\left\langle\mathcal{O}_{1} \cdots \mathcal{O}_{m} e^{-t \int \delta V}\right\rangle_{t+\Delta t}-\left\langle\mathcal{O}_{1} \cdots \mathcal{O}_{m} e^{-t \int \delta V}\right\rangle_{t}\right)
$$

where we have used $\left\langle\delta(\mathcal{O}) e^{-t \int \delta V}\right\rangle_{t}=0$. However, this will diverges if the vacuum at $t+\Delta t$ is not the vacuum at $t$. Now we assume the smoothness of changing the parameter $t .{ }^{12}$ Furthermore, we have seen that the moduli space of vacua of the theory at $t \rightarrow \infty$ is discrete. Thus, the vacuum is independent of $t$, which means that $\frac{\partial}{\partial t}\left\langle\mathcal{O}_{1} \cdots \mathcal{O}_{m} e^{-t \int \delta V}\right\rangle_{t}=$ 0 . Therefore, the gaugino condensation (3.17), which is correct value [33], is valid at $t=0$, i.e. the original theory.

\footnotetext{
${ }^{12}$ Although it is expected to be valid from the many examples using the localization technique, this should be justified. Unfortunately, for general cases, we can not justify it in this paper. In our case here we know the correct result, thus this assumption will be valid.
} 


\section{Including chiral multiplets}

Let us consider the chiral multiplets. By the regulator action for the vector multiplets, the effective gauge coupling determined by $t$ can be arbitrary weak. Thus, without introducing the regulator action for the chiral multiplets, the chiral multiplets can be integrated out first where the vector multiplets as the background fields. Indeed, for the (massive) SUSY QCD, the matters can be integrated out first trivially and the dynamical scale of the resulting SUSY Yang-Mills theory is computed by the usual way. Then, we can easily see that the resulting gaugino condensation is correct one.

If there is interaction terms in the superpotential, the effective superpotential after integrating out the chiral multiplets is non-trivial function of $S=\frac{1}{32 \pi^{2}} W_{\alpha} W^{\alpha}$. This is the case for the Dijkgraaf-Vafa conjecture [44-46]. In order to evaluate the gaugino condensation, we need to take into account not only the fundamental monopoles, but general anti-self-dual configurations because of the interactions which are induced by the chiral multiplets. This would be rather difficult. It could be useful to introduce the regulator action for the chiral multiplets for this case. We hope to report further progress for this in near future.

\section{Acknowledgments}

S.T. would like to thank K. Hosomichi for his collaboration at the early stage of this project and many useful and important comments and discussions. S.T. would like to thank G. Ishiki and M. Taki also for useful discussions. S.T. would like to thank E. Poppitz for useful comments about the 1-loop determinant. The work of S.T. is partly supported by the Japan Ministry of Education, Culture, Sports, Science and Technology (MEXT).

\section{A $\quad 4 \mathrm{~d} \mathcal{N}=1$ SUSY gauge theory on $S^{4}$}

In this appendix, we will explicitly construct the SUSY transformations and the SUSY invariant actions for $4 \mathrm{~d} \mathcal{N}=1$ SUSY gauge theories on $S^{4} .{ }^{13}$ However, as we will see in later, it may be impossible to construct a SUSY exact regulator term with a (semi)-positive definite bosonic part because $\left(\delta_{\xi}\right)^{2}$ can not be real nor pure imaginary as shown in [47].

First, we will construct $\mathcal{N}=1$ SUSY theories from the $\mathcal{N}=2$ SUSY theories on $S^{4}$, which are realized by a form given in $[6,20]$, by a truncation of the fields. The notation in this section is the one used in $[14,20]$. Especially, the indices $\mu, \nu, \cdots$ runs from 1 to 4 .

The metric of $S^{4}$ is taken to be

$$
d s_{S^{4}}^{2}=\ell^{2}\left(d \theta^{2}+\sin ^{2} \theta d s_{S^{3}}^{2}\right)=\frac{d r^{2}+r^{2} d s_{S^{3}}^{2}}{\left(1+\frac{r^{2}}{4 \ell^{2}}\right)^{2}}=\frac{\sum d x_{n}^{2}}{\left(1+\frac{r^{2}}{4 \ell^{2}}\right)^{2}},
$$

where $r^{2}=\sum_{n=1}^{4}\left(x^{n}\right)^{2}$ and we find $e^{a}=f \delta_{n}^{a} d x^{n}$ with $f \equiv\left(1+\frac{r^{2}}{4 \ell^{2}}\right)^{-1}$. We can embed the $S^{4}$ in $R^{5}$ as $Y_{1}^{2}+\cdots+Y_{5}^{2}=l^{2}$. The relation between $x^{n}$ and $Y^{n}(n=1, \ldots, 4)$ is $Y_{n}=\frac{x^{n}}{1+\frac{r^{2}}{4 l^{2}}}$.

\footnotetext{
${ }^{13}$ For the chiral multiplets, they were already explicitly represented in [19].
} 
We assume the following Killing Spinor equation:

$$
D_{\mu} \xi_{I}=\Gamma_{\mu} \tilde{\xi}_{I}
$$

Using the traceless $2 \times 2$ matrix $t_{I}^{J}$, which satisfies

$$
\left(t^{2}\right)_{I}^{J}=\frac{1}{4 l^{2}} \delta_{I}^{J}
$$

the Killing Spinor equation is solved by

$$
\xi_{I}=\frac{1}{\sqrt{1+\frac{r^{2}}{4 l^{2}}}}\left(\epsilon_{I}+x^{i} \Gamma_{i} \Gamma_{5} t_{I}^{J} \epsilon_{J}\right),
$$

where $i, j=1, \ldots, 4$ which are $4 \mathrm{~d}$ flat indices and $\epsilon_{I}, \epsilon_{I}^{\prime}$ are constants, and

$$
\tilde{\xi}_{I}=t_{I}^{J} \Gamma_{5} \xi_{J} .
$$

Therefore, by the $\mathrm{SU}(2)_{R}$ transformation, we will choose $\mathrm{e}^{14}$

$$
t_{I}^{J}=\frac{1}{2 l}\left(\sigma_{1}\right)_{I}^{J} .
$$

For the $\mathcal{N}=2$ vector multiplets. the $\mathcal{N}=2$ SUSY variation of fields on $S^{4}$ was given by

$$
\begin{aligned}
\delta_{\xi} A_{m} & =i \epsilon^{I J} \xi_{I} \Gamma_{m} \lambda_{J}, \\
\delta_{\xi} \sigma & =i \epsilon^{I J} \xi_{I} \lambda_{J}, \\
\delta_{\xi} \lambda_{I} & =-\frac{1}{2} \Gamma^{m n} \xi_{I} F_{m n}+\Gamma^{m} \xi_{I} D_{m} \sigma+\xi_{J} D_{K I} \epsilon^{J K}+2 \tilde{\xi}_{I} \sigma, \\
\delta_{\xi} D_{I J} & =-i\left(\xi_{I} \Gamma^{m} D_{m} \lambda_{J}+\xi_{J} \Gamma^{m} D_{m} \lambda_{I}\right)+\left[\sigma, \xi_{I} \lambda_{J}+\xi_{J} \lambda_{I}\right]+2 i \xi_{K} t_{I J} \Gamma_{5} \lambda^{K},
\end{aligned}
$$

where $m=1, \ldots, 5, A_{5}$ is a scalar, $F_{\mu 5}=D_{\mu} A_{5}$ and $D_{5}(*)=-i\left[*, A_{5}\right]$. Note that the $\mathrm{SU}(2)_{R}$ transformation including $t_{I}^{J}$ acts covariantly on the SUSY transformation. It was shown that the commutator of the two SUSY generators is a sum of a translation $\left(v^{m}\right)$, a gauge transformation $\left(\gamma+i v^{m} A_{m}\right)$, a dilation $(\rho)$, an R-rotation $\left(R_{I J}\right)$ and a Lorentz rotation $\left(\Theta^{a b}\right)$ :

$$
\begin{aligned}
{\left[\delta_{\xi}, \delta_{\eta}\right] A_{m} } & =-i v^{n} F_{n m}+D_{m} \gamma, \\
{\left[\delta_{\xi}, \delta_{\eta}\right] \sigma } & =-i v^{n} D_{n} \sigma+\rho \sigma, \\
{\left[\delta_{\xi}, \delta_{\eta}\right] \lambda_{I} } & =-i v^{n} \nabla_{n} \lambda_{I}+i\left[\gamma, \lambda_{I}\right]+\frac{3}{2} \rho \lambda_{I}+R_{I}^{\prime}{ }_{I} \lambda_{J}+\frac{1}{4} \Theta^{a b} \Gamma^{a b} \lambda \\
& =-i v^{n} D_{n} \lambda_{I}+i\left[\gamma, \lambda_{I}\right]+\frac{3}{2} \rho \lambda_{I}+R_{I}^{J} \lambda_{J}+\frac{1}{4} \Theta^{a b} \Gamma^{a b} \lambda, \\
{\left[\delta_{\xi}, \delta_{\eta}\right] D_{I J} } & =-i v^{n} \nabla_{n} D_{I J}+i\left[\gamma, D_{I J}\right]+2 \rho D_{I J}+R_{I}^{\prime K} D_{K J}+R_{J}^{\prime K} D_{I K} \\
& =-i v^{n} D_{n} D_{I J}+i\left[\gamma, D_{I J}\right]+2 \rho D_{I J}+R_{I}^{K} D_{K J}+R_{J}^{K} D_{I K},
\end{aligned}
$$

\footnotetext{
${ }^{14}$ In [14, 20], $t \sim \sigma_{3}$ was chosen. Our choice here is more convenient for $\mathcal{N}=1$ SUSY case.
} 
where $R_{I}^{J}=\epsilon^{J K} R_{I K}$ and

$$
\begin{aligned}
v^{m} & =2 \epsilon^{I J} \xi_{I} \Gamma^{m} \eta_{J}, \\
\gamma & =-2 i \epsilon^{I J} \xi_{I} \eta_{J} \sigma, \\
\rho & =-2 i \epsilon^{I J}\left(\xi_{I} \tilde{\eta}_{J}-\eta_{I} \tilde{\xi}_{J}\right)=0, \\
R_{I J} & =4 i t_{I J} \epsilon^{K L} \xi_{K} \Gamma_{5} \eta_{L}, \\
\Theta^{a b} & =-2 i \epsilon^{I J}\left(\tilde{\xi}_{I} \Gamma^{a b} \eta_{J}-\tilde{\eta}_{I} \Gamma^{a b} \xi_{J}\right) .
\end{aligned}
$$

Now let us consider the hypermultiplets, The system of $r$ hypermultiplets consists of scalars $q_{I}^{A}$, fermions $\psi^{A}$ and auxiliary scalars $F_{I}^{A}$. Here, $I=1,2$ is the $\mathrm{SU}(2) \mathrm{R}$-symmetry index and $A=1, \cdots, 2 r$. The fields obey the reality conditions

$$
\left(q_{I}^{A}\right)^{*}=\Omega_{A B} \epsilon^{I J} q_{J}^{B}, \quad\left(\psi^{A \alpha}\right)^{*}=\Omega_{A B} C_{\alpha \beta} \psi^{B \beta}, \quad\left(F_{I}^{A}\right)^{*}=\Omega_{A B} \epsilon^{I J} F_{J}^{B},
$$

where $\epsilon^{I J}, C_{\alpha \beta}, \Omega_{A B}$ are antisymmetric invariant tensors of $\mathrm{SU}(2) \simeq \mathrm{Sp}(1), \operatorname{Spin}(5) \simeq \mathrm{Sp}(2)$ and the "flavor symmetry" of $r$ free hypermultiplets $\operatorname{Sp}(r)$. The coupling to vector multiplets can be introduced via gauging a subgroup of $\operatorname{Sp}(r)$. In the Euclidian signature, we regard the fields are holomorphic variables, and then we will forget these reality conditions. (Note that two complex fields with a reality condition have two real components and two holomorphic fields without conditions also have two components.) To introduce the coupling to gauge fields and other fields in the vector multiplet, we need first to introduce the covariant derivative

$$
D_{m} \psi^{A} \equiv \partial_{m} \psi^{A}-i\left(A_{m}\right)_{B}^{A} \psi^{B}, \quad \text { etc. }
$$

Requiring $\Omega_{A B}$ to be gauge-invariant, one finds $\left(A_{m}\right)_{A B} \equiv \Omega_{A C}\left(A_{m}\right)_{B}^{C}$ to be symmetric in the indices $A, B$.

The $\mathcal{N}=2$ SUSY transformation was given by

$$
\begin{aligned}
\delta q_{I} & =-2 i \xi_{I} \psi, \\
\delta \psi & =\epsilon^{I J} \Gamma^{m} \xi_{I} D_{m} q_{J}+i \epsilon^{I J} \xi_{I} \sigma q_{J}+2 \epsilon^{I J} \tilde{\xi}_{I} q_{J}+\epsilon^{I^{\prime} J^{\prime}} \check{\xi}_{I^{\prime}} F_{J^{\prime}}, \\
\delta F_{I^{\prime}} & =2 \check{\xi}_{I^{\prime}}\left(i \Gamma^{m} D_{m} \psi+\sigma \psi+\epsilon^{K L} \lambda_{K} q_{L}\right) .
\end{aligned}
$$

Here, $\check{\xi}_{I^{\prime}}$ is a constant spinor which satisfies

$$
\epsilon^{I J} \xi_{I} \xi_{J}=\epsilon^{I^{\prime} J^{\prime}} \check{\xi}_{I^{\prime}} \check{\xi}_{J^{\prime}}, \quad \xi_{I} \check{\xi}_{J^{\prime}}=0, \quad \epsilon^{I J} \xi_{I} \Gamma^{m} \xi_{J}+\epsilon^{I^{\prime} J^{\prime}} \check{\xi}_{I^{\prime}} \Gamma^{m} \check{\xi}_{J^{\prime}}=0 .
$$

The square of $\delta$ is

$$
\begin{aligned}
\delta^{2} q_{I} & =i v^{m} D_{m} q_{I}-i \gamma q_{I}-R_{I}^{J} q_{J} \\
\delta^{2} \psi & =i v^{m} D_{m} \psi-i \gamma \psi-\frac{1}{4} \Theta^{a b} \Gamma^{a b} \psi \\
\delta^{2} F_{I^{\prime}} & =i v^{m} D_{m} F_{I^{\prime}}-i \gamma F_{I^{\prime}}+R_{I^{\prime}}^{J^{\prime}} F_{J^{\prime}},
\end{aligned}
$$


where

$$
\begin{aligned}
v^{m} & =\epsilon^{I J} \xi_{I} \Gamma^{m} \xi_{J}, \\
\gamma & =-i \epsilon^{I J} \xi_{I} \xi_{J} \sigma, \\
R_{I J} & =2 i\left(\epsilon^{K L} \xi_{K} \Gamma^{5} t_{I J} \xi_{L}\right), \\
\Theta^{a b} & =-2 i \epsilon^{I J} \tilde{\xi}_{I} \Gamma^{a b} \xi_{J}, \\
R_{I^{\prime} J^{\prime}}^{\prime} & =-2 i \check{\xi}_{I^{\prime}} \Gamma^{m} D_{m} \check{\xi}_{J^{\prime}},
\end{aligned}
$$

which is consistent with the one for the vectormultiplets.

Now we take a SUSY generator with a Killing spinor which satisfies ${ }^{15}$

$$
\Gamma_{5} \epsilon_{1}=\epsilon_{1}, \quad \Gamma_{5} \epsilon_{2}=-\epsilon_{2},
$$

which is equivalent to

$$
P \epsilon_{I}=\epsilon_{I},
$$

where $P \equiv \Gamma_{5}\left(\sigma_{3}\right)_{I}^{J}$. Then, we find

$$
\Gamma_{5} \xi_{1}=\xi_{1}, \quad \Gamma_{5} \xi_{2}=-\xi_{2}
$$

i.e. $P \xi_{I}=\xi_{I}$.

Because $\eta_{-} \xi_{+}=0$ for arbitrary chirality + and - spinors, these Killing spinors satisfy the followings:

$$
\epsilon^{I J} \xi_{I} \xi_{J}=0, \quad v^{5}=2 \xi_{1} \Gamma^{5} \xi_{2}=-2 \xi_{1} \xi_{2}=0, \quad R_{I J}=-4 t_{I J} \xi_{1} \xi_{2}=0 .
$$

This implies that we can take

$$
\check{\xi}_{I^{\prime}}=i \xi_{I}
$$

For this choice, $R_{I^{\prime} J^{\prime}}^{\prime}=0$.

For the scalars and vectors, we will define the action of $P$ as

$$
\begin{aligned}
P A_{\mu} & =A_{\mu}, \quad P D_{12}=D_{12}, \\
P A_{5} & =-A_{5}, \quad P \sigma=-\sigma, \quad P D_{11}=-D_{11}, \quad P D_{22}=-D_{22} .
\end{aligned}
$$

Then, we find that

$$
\left[\delta_{\xi}, P\right]=0
$$

This means that, schematically, $\delta_{\xi} \Phi_{+}=\Phi_{+}+\Phi_{+} \Phi_{+}+\Phi_{-} \Phi_{-}$and $\delta_{\xi} \Phi_{-}=\Phi_{-}+\Phi_{-} \Phi_{+}$.

Now, we consider only the $P=1$ fields as an $\mathcal{N}=1$ SUSY fields. The SUSY transformation on the $\mathcal{N}=1$ SUSY fields are defined just by taking $P=-1$ fields vanish in

\footnotetext{
${ }^{15}$ We can not impose the twisted Majorana condition for these.
} 
the SUSY transformation of $\mathcal{N}=2$ SUSY. Thus, the SUSY algebra is obtained by taking $P=-1$ fields vanish in the one for $\mathcal{N}=2$ SUSY, which is consistent.

Explicitly, we find

$$
\begin{aligned}
\delta_{\xi} A_{\mu} & =i\left(\xi_{1} \Gamma_{\mu} \lambda_{2}^{-}-\xi_{2} \Gamma_{\mu} \lambda_{1}^{+}\right), \\
\delta_{\xi} \lambda_{1}^{+} & =-\frac{1}{2} \Gamma^{\mu \nu} \xi_{1} F_{\mu \nu}+\xi_{1} D_{12}, \\
\delta_{\xi} \lambda_{2}^{-} & =-\frac{1}{2} \Gamma^{\mu \nu} \xi_{2} F_{\mu \nu}-\xi_{2} D_{12}, \\
\delta_{\xi} D_{12} & =-i \xi_{1} \Gamma^{\mu} D_{\mu} \lambda_{2}^{-}-i \xi_{2} \Gamma^{\mu} D_{\mu} \lambda_{1}^{+},
\end{aligned}
$$

where $\lambda_{1}^{+}=\left(1+\Gamma_{5}\right) / 2 \lambda_{1}$ and $\lambda_{2}^{-}=\left(1-\Gamma_{5}\right) / 2 \lambda_{2}$.

If we define $D \equiv D_{12}, \xi \equiv \xi_{1}+\xi_{2}$ and $\lambda \equiv \lambda_{1}^{+}+\lambda_{2}^{-}$, we find

$$
\begin{aligned}
\delta_{\xi} A_{\mu} & =-i \xi \Gamma_{\mu} \Gamma_{5} \lambda, \\
\delta_{\xi} \lambda & =-\frac{1}{2} \Gamma^{\mu \nu} \xi F_{\mu \nu}+\Gamma_{5} \xi D, \\
\delta_{\xi} D & =-i \xi \Gamma^{\mu} D_{\mu} \lambda,
\end{aligned}
$$

which is same as the one in flat space except that the vierbein and the connections are the ones on $S^{4}$.

For the hypermultiplets, we can take

$$
\Gamma_{5} \check{\xi}_{1}=\check{\xi}_{1}, \quad \Gamma_{5} \check{\xi}_{2}=-\check{\xi}_{2} .
$$

We will take

$$
\Omega_{A B}=i \sigma_{2} \oplus i \sigma_{2} \oplus \cdots \oplus i \sigma_{2}
$$

and we will change the notation for the index $A$, for example,

$$
\psi^{A} \rightarrow \psi^{A, i}
$$

now the $A=1, \ldots, r$ and $i=1,2$. Then, we define $P \psi^{A, i} \equiv \Gamma_{5}(-1)^{i+1} \psi^{A i}, P q_{I}^{A, i}=$ $(-1)^{I+i} q_{I}^{A, i}$ and $P F_{I}^{A, i}=-(-1)^{I+i} F_{I}^{A+i}$. With this, we can consistently truncate the $\mathcal{N}=2$ fields to the $P=1$ fields, i.e. $\mathcal{N}=1$ fields, and find

$$
\begin{aligned}
\delta q^{A, i} & =-2 i \xi_{i} \psi^{A, i}, \\
\delta \psi^{A, i} & =-\Gamma^{\mu} \epsilon^{i j} \xi_{j} D_{\mu} q^{A, i}-\frac{1}{l} \xi_{i} q^{A, i}+\check{\xi}_{i} F^{A, i}, \\
\delta F^{A, i} & =2 \epsilon^{i j} \check{\xi}_{j}\left(i \Gamma^{\mu} D_{\mu} \psi^{A, i}-\epsilon^{i k} \lambda_{k} q^{A, i}\right),
\end{aligned}
$$

where we have not summed over $i$ and we defined $q^{A, i} \equiv q_{i}^{A, i}$ (no summation for $i$ ), $F^{A, i} \equiv(-1)^{i-1} F_{3-i}^{A, i}$ and $\psi^{A, i} \equiv\left(1+(-1)^{i+1} \Gamma_{5}\right) / 2 \psi^{A, i}$. This form is slightly different from the one in [19]. For $\check{\xi}_{I^{\prime}}=i \xi_{I}$, however, if we define

$$
F^{\prime}=F+\frac{i}{l} q
$$

we obtain the same form. 
We will regard the fields as holomorphic and then forget the reality conditions. Indeed, we will not encounter any complex conjugate of the fields below.

Below we will try to construct SUSY invariant actions. We will drop the total divergent terms below for the notational convenience.

The $\mathcal{N}=2$ SUSY invariant action for vectormultiplets on $S^{4}$ is

$$
\begin{aligned}
\mathcal{L}_{S^{4}}^{\text {vector }}= & \frac{1}{2} F_{m n} F^{m n}-D_{m} \sigma D^{m} \sigma+i \lambda_{I} \Gamma^{m} D_{m} \lambda^{I}-\lambda_{I}\left[\sigma, \lambda^{I}\right] \\
& -\frac{1}{2}\left(D_{I J}-2 A_{5} t_{I J}\right)\left(D^{I J}-2 A_{5} t^{I J}\right)-4 t_{I J} t^{I J}\left(\left(A_{5}\right)^{2}-\sigma^{2}\right),
\end{aligned}
$$

where $\partial_{5}=0$, which is the usual SUSY Yang-Mills Lagrangian of the vector multiplet used in [2] with some field redefinitions [20]. This action does not have any terms linear in fields with $P=-1$. Thus, the following truncated action is invariant under the $\mathcal{N}=1$ SUSY:

$$
\mathcal{L}_{S^{4}}^{\text {vector }}=\frac{1}{2} F_{\mu \nu} F^{\mu \nu}+i \lambda_{1}^{+} \Gamma^{\mu} D_{\mu} \lambda_{2}^{-}-i \lambda_{2}^{-} \Gamma^{\mu} D_{\mu} \lambda_{1}^{+}-\left(D_{12}\right)^{2},
$$

which take the same form in the flat space.

Now we will consider the hypermultiplets. We have the $\mathcal{N}=2$ SUSY invariant Lagrangian on $S^{4}$ :

$$
\begin{aligned}
\mathcal{L}_{S^{4}}^{\text {hyper }}= & \epsilon^{I J}\left(D_{\mu} \bar{q}_{I} D^{\mu} q_{J}+\bar{q}_{I}\left(A_{5}\right)^{2} q_{J}-\bar{q}_{I} \sigma^{2} q_{J}\right)-2\left(i \bar{\psi} \Gamma^{\mu} D_{\mu} \psi+\bar{\psi} \Gamma_{5} A_{5} \psi+\bar{\psi} \sigma \psi\right) \\
& -i \bar{q}_{I} D^{\prime I J} q_{J}-4 \epsilon^{I J} \bar{\psi} \lambda_{I} q_{J}-\epsilon^{I^{\prime} J^{\prime}} \bar{F}_{I^{\prime}} F_{J^{\prime}} \\
& -8 t^{K L} t_{K L} \epsilon^{I J} \bar{q}_{I} q_{J}
\end{aligned}
$$

where, we have introduced the notation $\bar{\psi}_{B} \equiv \psi^{A} \Omega_{A B}$ and suppress the indices $A, B, \cdots$, such that

$$
\begin{aligned}
\epsilon^{I J} \Omega_{A B} D_{\mu} q_{I}^{A} D^{\mu} q_{J}^{B} & \equiv \epsilon^{I J} D_{\mu} \bar{q}_{I} D^{\mu} q_{J}, \\
\Omega_{A B} \psi^{A} \Gamma^{\mu}\left(A_{\mu}\right)_{C}^{B} \psi^{C} & \equiv \bar{\psi} \Gamma^{\mu} A_{\mu} \psi, \quad \text { etc. }
\end{aligned}
$$

Because this action does not have any term which is linear in the $P=-1$ fields, the following action is $\mathcal{N}=1$ SUSY invariant:

$$
\begin{aligned}
\mathcal{L}_{S^{4}}^{\text {chiral }}= & 2 h_{A B} D_{\mu} q^{A} D^{\mu} q^{B}-2 i \Omega_{A B} \bar{\psi}^{A} \Gamma^{\mu} D_{\mu} \psi^{B} \\
& -2 i h_{A B} q^{A} D^{12} q^{B}-4 h_{A B} \psi^{A}\left(\lambda_{A \bmod 2} q\right)^{B}-2 h_{A B} F^{A} F^{B}-16 t^{K L_{1}} t_{K L} h_{A B} q^{A} q^{B},
\end{aligned}
$$

where

$$
h_{A B} \equiv \Omega_{A B}(-1)^{A}=\sigma_{1} \oplus \sigma_{1} \oplus \cdots \oplus \sigma_{1} .
$$

We can easily see that the flat space superpotential terms written in $F^{\prime}=F+\frac{i}{l} q$ is invariant under the $\mathcal{N}=2$ SUSY on $S^{4}$ because the superpotential is gauge invariant and the $F^{\prime}$ enters in the superpotential at most linearly. Note that the SUSY transformation 
is modified only for $F^{\prime}$ except the modification of the metric and connections. The SUSY transformation $\check{\xi}_{2} \Gamma_{\mu} D_{\mu} \psi$ in $F^{\prime}$ gives an extra contribution $D_{\mu} \breve{\xi}_{2} \Gamma_{\mu} \psi$, which indeed cancel with the one from the modified term in $F^{\prime}$. The theta term is also $\mathcal{N}=1$ SUSY invariant because it is a topological term.

Let us consider SUSY invariant operators. Because $\xi_{1}=0$ at $x^{\mu}=0$, we find $\delta_{\xi} \lambda_{1}^{+}\left(x^{\mu}=\right.$ $0)=0, \delta_{\xi} q^{A, 1}\left(x^{\mu}=0\right)=0$, and $\delta_{\xi} F^{A, 2}\left(x^{\mu}=0\right)=0$. Similarly we find $\delta_{\xi} \lambda_{2}^{-}\left(x^{\mu}=\infty\right)=0$, $\delta_{\xi} q^{A, 2}\left(x^{\mu}=\infty\right)=0$, and $\delta_{\xi} F^{A, 1}\left(x^{\mu}=\infty\right)=0$.

Note that $q^{A, 2}$ can be considered as $\left(q^{A, 1}\right)^{\dagger}$, thus the lowest components of the chiral superfields inserted at the north pole and the lowest components of the anti-chiral superfields inserted at the south pole are the SUSY invariants operators. (In the flat case, it is clear that $\bar{D}^{2}\left(\Phi^{\dagger}\right) \sim \bar{F}$ is a chiral operator because $\bar{D}^{3}=0$.)

Now, we will try to apply the localization technique used in [2] to the $\mathcal{N}=1$ SUSY theory on $S^{4}$. However, as we will see below, it may be impossible to construct a term $\int_{S^{4}} \delta V$ which has a positive definite real part.

First we will try to construct $\int_{S^{4}} \delta V$ on $S^{4}$ with a appropriate properties. We take $\xi_{I}$ as Grassmann-even spinors such that $\delta_{\xi}$ is a fermionic transformation. We can easily see that $\xi_{1} \xi_{1}=0$, which is followed form $C^{T}=-C$ and $\xi_{1} \xi_{2}=0$ because $\Gamma_{5} \xi_{1}=\xi_{1}$ and $\Gamma_{5} \xi_{2}=-\xi_{2}$. In order to use the localization technique, we need a regulator Lagrangian $\delta_{\xi} V$ with $\int_{S^{4}}\left(\delta_{\xi}\right)^{2} V=0$. The usual choice is a form like $V=\operatorname{tr}\left[\left(\delta_{\xi} \lambda\right)^{\dagger} \lambda\right]$, where $\left(\delta_{\xi} \lambda\right)^{\dagger}$ should be defined using the holomorphic fields and $\xi_{I}$. However, if we assume the following form

$$
\left(\xi_{I}\right)^{\star}=\left(M^{I J}+N^{I J} \Gamma_{5}\right) C \xi_{J},
$$

where $M, N$ are arbitrary matrices, we find a contradiction. Indeed, the $\Gamma_{5}$-chirality requires that $M, N$ are diagonal, and we can assume $\left(\xi_{I}\right)^{\star}=M C \xi_{I}$. Then, we find $\xi_{I}=M^{*} C^{*}\left(\xi_{I}\right)^{*}=M^{*} C^{*} M C \xi_{I}=-|M|^{2} \xi_{I}$, which means $\xi_{I}=0$. We can use a tensor satisfying $\mathcal{L}_{v} T=0$, however, as far as we have checked, there is no localization terms with a positive definite real part of bosonic terms. These difficulty will be originate from the nonzero complex value of the $v^{\mu}=\xi_{I} \Gamma^{\mu} \xi^{I}$ even if we take $\epsilon_{1}=0$.

Open Access. This article is distributed under the terms of the Creative Commons Attribution License (CC-BY 4.0), which permits any use, distribution and reproduction in any medium, provided the original author(s) and source are credited.

\section{References}

[1] E. Witten, Constraints on Supersymmetry Breaking, Nucl. Phys. B 202 (1982) 253 [INSPIRE].

[2] V. Pestun, Localization of gauge theory on a four-sphere and supersymmetric Wilson loops, Commun. Math. Phys. 313 (2012) 71 [arXiv:0712.2824] [INSPIRE].

[3] E. Witten, Topological Quantum Field Theory, Commun. Math. Phys. 117 (1988) 353 [INSPIRE].

[4] N.A. Nekrasov, Seiberg-Witten prepotential from instanton counting, Adv. Theor. Math. Phys. 7 (2004) 831 [hep-th/0206161] [INSPIRE]. 
[5] N. Hama and K. Hosomichi, Seiberg-Witten Theories on Ellipsoids, JHEP 09 (2012) 033 [Addendum ibid. 10 (2012) 051] [arXiv:1206.6359] [INSPIRE].

[6] T. Nosaka and S. Terashima, Supersymmetric Gauge Theories on a Squashed Four-Sphere, JHEP 12 (2013) 001 [arXiv:1310.5939] [INSPIRE].

[7] Y. Imamura, Relation between the $4 d$ superconformal index and the $S^{3}$ partition function, JHEP 09 (2011) 133 [arXiv:1104.4482] [INSPIRE].

[8] A. Kapustin, B. Willett and I. Yaakov, Exact Results for Wilson Loops in Superconformal Chern-Simons Theories with Matter, JHEP 03 (2010) 089 [arXiv:0909.4559] [INSPIRE].

[9] D.L. Jafferis, The Exact Superconformal R-Symmetry Extremizes Z, JHEP 05 (2012) 159 [arXiv: 1012.3210] [INSPIRE].

[10] N. Hama, K. Hosomichi and S. Lee, Notes on SUSY Gauge Theories on Three-Sphere, JHEP 03 (2011) 127 [arXiv: 1012.3512] [INSPIRE].

[11] N. Hama, K. Hosomichi and S. Lee, SUSY Gauge Theories on Squashed Three-Spheres, JHEP 05 (2011) 014 [arXiv:1102.4716] [INSPIRE].

[12] F. Benini and S. Cremonesi, Partition functions of $\mathcal{N}=(2,2)$ gauge theories on $S^{2}$ and vortices, Commun. Math. Phys. 334 (2015) 1483 [arXiv:1206.2356] [INSPIRE].

[13] N. Doroud, J. Gomis, B. Le Floch and S. Lee, Exact Results in D $=2$ Supersymmetric Gauge Theories, JHEP 05 (2013) 093 [arXiv: 1206.2606] [INSPIRE].

[14] K. Hosomichi, R.-K. Seong and S. Terashima, Supersymmetric Gauge Theories on the Five-Sphere, Nucl. Phys. B 865 (2012) 376 [arXiv:1203.0371] [INSPIRE].

[15] J. Källén and M. Zabzine, Twisted supersymmetric 5D Yang-Mills theory and contact geometry, JHEP 05 (2012) 125 [arXiv:1202.1956] [INSPIRE].

[16] H.-C. Kim and S. Kim, M5-branes from gauge theories on the 5-sphere, JHEP 05 (2013) 144 [arXiv:1206.6339] [INSPIRE].

[17] T. Kawano and N. Matsumiya, 5D SYM on 3D Sphere and $2 D Y M$, Phys. Lett. B 716 (2012) 450 [arXiv:1206.5966] [INSPIRE].

[18] J. Källén, J. Qiu and M. Zabzine, The perturbative partition function of supersymmetric $5 D$ Yang-Mills theory with matter on the five-sphere, JHEP 08 (2012) 157 [arXiv:1206.6008] [INSPIRE].

[19] G. Festuccia and N. Seiberg, Rigid Supersymmetric Theories in Curved Superspace, JHEP 06 (2011) 114 [arXiv: 1105.0689] [INSPIRE].

[20] S. Terashima, Supersymmetric gauge theories on $S^{4} \times S^{1}$, Phys. Rev. D 89 (2014) 125001 [arXiv: 1207.2163] [INSPIRE].

[21] Y. Asano, G. Ishiki, T. Okada and S. Shimasaki, Exact results for perturbative partition functions of theories with $\mathrm{SU}(2 \mid 4)$ symmetry, JHEP 02 (2013) 148 [arXiv:1211.0364] [INSPIRE].

[22] S. Sugishita and S. Terashima, Exact Results in Supersymmetric Field Theories on Manifolds with Boundaries, JHEP 11 (2013) 021 [arXiv: 1308.1973] [INSPIRE].

[23] K.A. Intriligator and N. Seiberg, Lectures on supersymmetric gauge theories and electric-magnetic duality, Nucl. Phys. Proc. Suppl. 45BC (1996) 1 [hep-th/9509066] [INSPIRE]. 
[24] N. Seiberg, Exact results on the space of vacua of four-dimensional SUSY gauge theories, Phys. Rev. D 49 (1994) 6857 [hep-th/9402044] [INSPIRE].

[25] I. Affleck, M. Dine and N. Seiberg, Dynamical Supersymmetry Breaking in Four-Dimensions and Its Phenomenological Implications, Nucl. Phys. B 256 (1985) 557 [InSPIRE].

[26] V.A. Novikov, M.A. Shifman, A.I. Vainshtein and V.I. Zakharov, Supersymmetric Instanton Calculus (Gauge Theories with Matter), Nucl. Phys. B 260 (1985) 157 [Yad. Fiz. 42 (1985) 1499] [INSPIRE].

[27] N. Seiberg and E. Witten, Electric-magnetic duality, monopole condensation and confinement in $N=2$ supersymmetric Yang-Mills theory, Nucl. Phys. B 426 (1994) 19 [Erratum ibid. B 430 (1994) 485] [hep-th/9407087] [INSPIRE].

[28] F.A.H. Dolan, V.P. Spiridonov and G.S. Vartanov, From 4d superconformal indices to $3 d$ partition functions, Phys. Lett. B 704 (2011) 234 [arXiv:1104.1787] [INSPIRE].

[29] A. Gadde and W. Yan, Reducing the $4 d$ Index to the $S^{3}$ Partition Function, JHEP 12 (2012) 003 [arXiv:1104.2592] [INSPIRE].

[30] D. Amati, K. Konishi, Y. Meurice, G.C. Rossi and G. Veneziano, Nonperturbative Aspects in Supersymmetric Gauge Theories, Phys. Rept. 162 (1988) 169 [INSPIRE].

[31] N.M. Davies, T.J. Hollowood, V.V. Khoze and M.P. Mattis, Gluino condensate and magnetic monopoles in supersymmetric gluodynamics, Nucl. Phys. B 559 (1999) 123 [hep-th/9905015] [INSPIRE].

[32] N.M. Davies and V.V. Khoze, On Affleck-Dine-Seiberg superpotential and magnetic monopoles in supersymmetric QCD, JHEP 01 (2000) 015 [hep-th/9911112] [INSPIRE].

[33] N.M. Davies, T.J. Hollowood and V.V. Khoze, Monopoles, affine algebras and the gluino condensate, J. Math. Phys. 44 (2003) 3640 [hep-th/0006011] [INSPIRE].

[34] B. Assel, D. Cassani and D. Martelli, Localization on Hopf surfaces, JHEP 08 (2014) 123 [arXiv: 1405.5144] [INSPIRE].

[35] K. Hosomichi, A review on SUSY gauge theories on $S^{3}$, arXiv:1412.7128 [INSPIRE].

[36] C. Romelsberger, Counting chiral primaries in $N=1, d=4$ superconformal field theories, Nucl. Phys. B $\mathbf{7 4 7}$ (2006) 329 [hep-th/0510060] [INSPIRE].

[37] J. Kinney, J.M. Maldacena, S. Minwalla and S. Raju, An Index for 4 dimensional super conformal theories, Commun. Math. Phys. 275 (2007) 209 [hep-th/0510251] [INSPIRE].

[38] M.A. Shifman and A.I. Vainshtein, On Gluino Condensation in Supersymmetric Gauge Theories. $\mathrm{SU}(N)$ and $O(N)$ Groups, Nucl. Phys. B 296 (1988) 445 [Sov. Phys. JETP 66 (1987) 1100] [INSPIRE].

[39] A.Y. Morozov, M.A. Olshanetsky and M.A. Shifman, Gluino Condensate in Supersymmetric Gluodynamics (II), Nucl. Phys. B 304 (1988) 291 [Sov. Phys. JETP 67 (1988) 222] [Zh. Eksp. Teor. Fiz. 94 (1988) 18] [INSPIRE].

[40] D. Finnell and P. Pouliot, Instanton calculations versus exact results in four-dimensional SUSY gauge theories, Nucl. Phys. B 453 (1995) 225 [hep-th/9503115] [InSPIRE].

[41] E. Poppitz, T. Schäfer and M. Ünsal, Continuity, Deconfinement and (Super) Yang-Mills Theory, JHEP 10 (2012) 115 [arXiv: 1205. 0290] [INSPIRE]. 
[42] M.M. Anber, E. Poppitz and B. Teeple, Deconfinement and continuity between thermal and (super) Yang-Mills theory for all gauge groups, JHEP 09 (2014) 040 [arXiv:1406.1199] [INSPIRE].

[43] K.-M. Lee and P. Yi, Monopoles and instantons on partially compactified D-branes, Phys. Rev. D 56 (1997) 3711 [hep-th/9702107] [INSPIRE].

[44] R. Dijkgraaf and C. Vafa, Matrix models, topological strings and supersymmetric gauge theories, Nucl. Phys. B 644 (2002) 3 [hep-th/0206255] [INSPIRE].

[45] R. Dijkgraaf and C. Vafa, On geometry and matrix models, Nucl. Phys. B 644 (2002) 21 [hep-th/0207106] [INSPIRE].

[46] R. Dijkgraaf and C. Vafa, A Perturbative window into nonperturbative physics, hep-th/0208048 [INSPIRE].

[47] E. Gerchkovitz, J. Gomis and Z. Komargodski, Sphere Partition Functions and the Zamolodchikov Metric, JHEP 11 (2014) 001 [arXiv:1405.7271] [INSPIRE]. 